\title{
Effect of Growth Hormone Therapy on Height Velocity in Korean Children with Idiopathic Short Stature: A Phase III Randomised Controlled Trial
}

\author{
Woo Yeong Chung ${ }^{a}$ Han-Wook Yoo ${ }^{b}$ Jin Soon Hwang ${ }^{c}$ Cheol Woo Ko ${ }^{d}$ \\ Ho-Seong Kim ${ }^{e}$ Dong-Kyu Jin ${ }^{f}$ Kee-Hyoung Leeg Heon-Seok Han ${ }^{\text {h }}$ \\ Premila Paranchothy ${ }^{i}$ Byung-Kyu Suh ${ }^{j}$ \\ a Department of Pediatrics, Inje University Busan Paik Hospital, Busan, South Korea; ${ }^{b}$ Department of Pediatrics, \\ Asan Medical Centre, University of Ulsan College of Medicine, Seoul, South Korea; ' ${ }^{\circ}$ Department of Pediatrics, \\ Ajou University Hospital, Ajou University School of Medicine, Suwon, South Korea; ${ }^{d}$ Department of Pediatrics, \\ Kyungpook National University Hospital, Kyungpook National University School of Medicine, Daegu, South Korea; \\ e Department of Pediatrics, Severance Children's Hospital, Yonsei University College of Medicine, Seoul, \\ South Korea; ${ }^{f}$ Department of Pediatrics, Samsung Medical Centre, Sungkyunkwan University School of Medicine, \\ Seoul, South Korea; ${ }^{9}$ Department of Pediatrics, Korea University Anam Hospital, Korea University College of

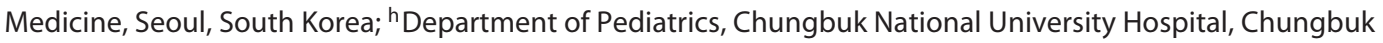 \\ National University College of Medicine, Cheongju, South Korea; 'Merck Ltd, Korea, Seoul, South Korea; \\ 'Department of Pediatrics, College of Medicine, The Catholic University of Korea, Seoul, South Korea
}

\section{Keywords}

Idiopathic short stature $\cdot$ Recombinant human growth hormone · Height velocity · Efficacy · Safety

\footnotetext{
Abstract

Background/Aims: The SYNERGY (Saizen ${ }^{\circledR}$ for Your New Life and Brighter Tomorrow without Growth Deficiency) study is the first randomised multi-centre, open-label study to assess the short-term efficacy and safety of this recombinant human growth hormone $(\mathrm{r}-\mathrm{hGH})$ preparation for prepubertal children with idiopathic short stature in South Korea. Methods: The SYNERGY study (ClinicalTrials.gov NCT01746862) was conducted at 9 centres throughout South Korea between December 2012 and March 2015. The primary endpoint was difference in height velocity from baseline to 6
}

months in the treatment and control arms. Results: 97 children were screened; 90 were randomly assigned: 60 children to $0.067 \mathrm{mg} / \mathrm{kg} /$ day $\mathrm{r}$-hGH for 12 months (treatment) and 30 children to 6 months of no treatment followed by $0.067 \mathrm{mg} /$ $\mathrm{kg} /$ day $\mathrm{r}$-hGH for 6 months (control). The 6-month mean height velocity in the treatment group increased from 5.63 $\mathrm{cm} /$ year (SD 1.62) to $10.08 \mathrm{~cm} /$ year (SD 1.92) $(p<0.0001)$ and from $4.94 \mathrm{~cm} /$ year (SD 1.91) to $5.92 \mathrm{~cm} /$ year (SD 2.01) $(p=$ 0.0938 ) in the control group (between-group difference 3.47 $\mathrm{cm} /$ year, 95\% Cl 2.17-4.78; $p<0.0001$ ). Adherence was $>90 \%$ throughout the study. The safety profile was consistent with that already known for $\mathrm{r}-\mathrm{hGH}$. Conclusion: Treatment with $\mathrm{r}$-hGH in the SYNERGY study demonstrated a statistically significant increase in height velocity at 6 months.

(C) 2018 The Author(s)

Published by S. Karger AG, Basel

\begin{tabular}{ll}
\hline KARGER & $\begin{array}{l}\text { @ 2018 The Author(s) } \\
\text { Published by S. Karger AG, Basel Openger }\end{array}$ \\
E-Mail karger@karger.com & This article is licensed under the Creative Commons Attribution- \\
www.karger.com/hrp & $\begin{array}{l}\text { NonCommercial-NoDerivatives 4.0 International License (CC BY- } \\
\text { NC-ND) (http://www.karger.com/Services/OpenAccessLicense). } \\
\text { Usage and distribution for commercial purposes as well as any dis- } \\
\text { tribution of modified material requires written permission. }\end{array}$
\end{tabular}

Prof. Byung-Kyu Suh

Department of Pediatrics, College of Medicine

The Catholic University of Korea

\#222 Banpo-daero Seocho-gu, Seoul 06591 (South Korea)

E-Mailsuhbk@ catholic.ac.kr 
Short stature [1] and growth failure [2,3] are some of the most common causes for referral to paediatric endocrinology clinics, general paediatricians, and primary health care physicians [3].

Although many of the causes of short stature and growth failure have been identified, children who fulfil the criteria for these disorders but who have no evidence of systemic, endocrine, nutritional, or chromosomal abnormalities, and who have normal simulated growth hormone $(\mathrm{GH})$ concentrations, are commonly diagnosed under the umbrella term of idiopathic short stature (ISS). The causes of ISS are likely to be heterogeneous and related to subtle disorders of GH secretion or sensitivity or a combination of genetic factors that influence growth plate biology, and are unlikely to be picked up during conventional diagnostic workup $[4,5]$. Even with the advent of comprehensive molecular studies, the diagnosis of ISS remains based on auxological assessment (height below -2 standard deviation scores [SDS]) and the exclusion of other causes [6].

Children with ISS are of normal size at birth but grow slowly during early childhood, meaning their height generally falls within the range for ISS by the time they start school [3]. In the long term, untreated children with ISS will usually be shorter than their predicted adult heights and significantly below their genetic target height, potentially resulting in detrimental effects on quality of life, necessitating early identification and treatment [7-11].

Treatment of ISS with somatropin (recombinant human GH $[\mathrm{r}-\mathrm{hGH}]$ ) was approved by the United States Food and Drug Administration (US FDA) in 2003, and several formulations of $\mathrm{r}$-hGH have shown short-term efficacy (improvements in height velocity) and long-term efficacy (attainment of near-adult height) in children with ISS $[4,6,12,13]$. In South Korea, the $\mathrm{r}$-hGH preparation Saizen ${ }^{\circledR}$ (Merck KGaA, Darmstadt, Germany) is approved for the treatment of children with growth failure associated with GH deficiency, chronic renal failure, Turner syndrome and for children born small for gestational age. Saizen ${ }^{\circledR}$ is not currently approved for ISS in South Korea.

The SYNERGY (Saizen ${ }^{\circledR}$ for Your New Life and Brighter Tomorrow without Growth Deficiency) study is the first randomised multi-centre, open-label study to assess the short-term efficacy and safety of r-hGH for children with ISS in South Korea. The aim of the SYNERGY study is to expand the indication of this preparation of $\mathrm{r}$-hGH as an additional option for the treatment of children with ISS in Korea.

Growth Hormone in Korean Children with ISS

\section{Methods}

\section{Study Participants}

The SYNERGY study (ClinicalTrials.gov NCT01746862) was a 12-month, open-label, randomised, two-arm, parallel group, delayed-treatment group-controlled ( 6 months of no treatment followed by 6 months of treatment with r-hGH $0.067 \mathrm{mg} / \mathrm{kg} / \mathrm{day}, 6$ days per week) phase III study. SYNERGY was conducted at 9 centres throughout South Korea between December 2012 and March 2015. The aim of SYNERGY was to assess the efficacy and safety of treatment with $\mathrm{r}-\mathrm{hGH}$ in prepubertal children with ISS.

Children were eligible for inclusion if they had official height records available and were $\geq 5$ years of age; were prepubertal; had height $<3$ rd percentile for the Korean National Growth Charts for the same age and sex; had peak serum GH $>10 \mu \mathrm{g} / \mathrm{L}$ in at least 2 $\mathrm{GH}$ stimulation tests; were naïve to $\mathrm{r}-\mathrm{hGH}$ therapy; had a gestational age $>34$ weeks and an appropriate birth weight, normal thyroid function and karyotype (girls); and had bone age $\leq 9$ years in girls and $\leq 10$ years in boys with no more than 3 years' difference from chronological age. Children were excluded if they were diagnosed with predesignated pathological processes. Children were also excluded if they entered puberty (testicular volume $\geq 4 \mathrm{~mL}$ in boys or breast Tanner stage $\geq 2$ in girls) during their participation in the study.

The SYNERGY study was conducted in compliance with the clinical study protocol, Good Clinical Practice, and the applicable Korean regulatory requirements. The institutional review board at each study site provided favourable opinion/approval, and the principal investigators were responsible for the conduct of the study. Written assent of the child and written informed consent of the parent/guardian to participate in the study were obtained before any study-related activities were carried out.

\section{Study Procedures}

Following informed consent, screening-related procedures were carried out during a period of up to 28 days. Eligible children were then randomly assigned in a 2:1 ratio, using a permuted block randomisation method based on the order of recruitment at each participating centre, to receive $0.067 \mathrm{mg} / \mathrm{kg}$ /day $\mathrm{r}-\mathrm{hGH}$ for 12 months (treatment group) or no treatment for 6 months followed by 6 months of treatment with $\mathrm{r}-\mathrm{hGH} 0.067 \mathrm{mg} / \mathrm{kg} /$ day (control group). Randomisation codes were generated centrally by a contract research organisation. No masking to treatment allocation was carried out.

The primary endpoint of SYNERGY was the change in height velocity from baseline to 6 months between the treatment and the control groups. Secondary endpoints were the between-group change in height velocity at 12 months; height and height SDS; IGF- 1 and IGFBP- 3 concentrations at 6 and 12 months; treatment adherence; and overall safety profile.

At each centre, children were assessed at weeks 13, 26, 39, and 52 for auxological parameters (e.g., height and weight measurements), potential adverse events (AEs), dispensing of the study drug and diary entries (to evaluate adherence and concomitant medications), and review of the techniques for reconstitution using click easy ${ }^{\circledR}$ and injection using easypod ${ }^{\circledR}$. An additional visit was conducted at 56 weeks to assess any AEs that may have developed or persisted since the last dose of $\mathrm{r}-\mathrm{hGH}$. Radiological evaluation of bone age [14] was undertaken at screening and at weeks 26 and 52 by an independent investigator blinded to treatment group and 


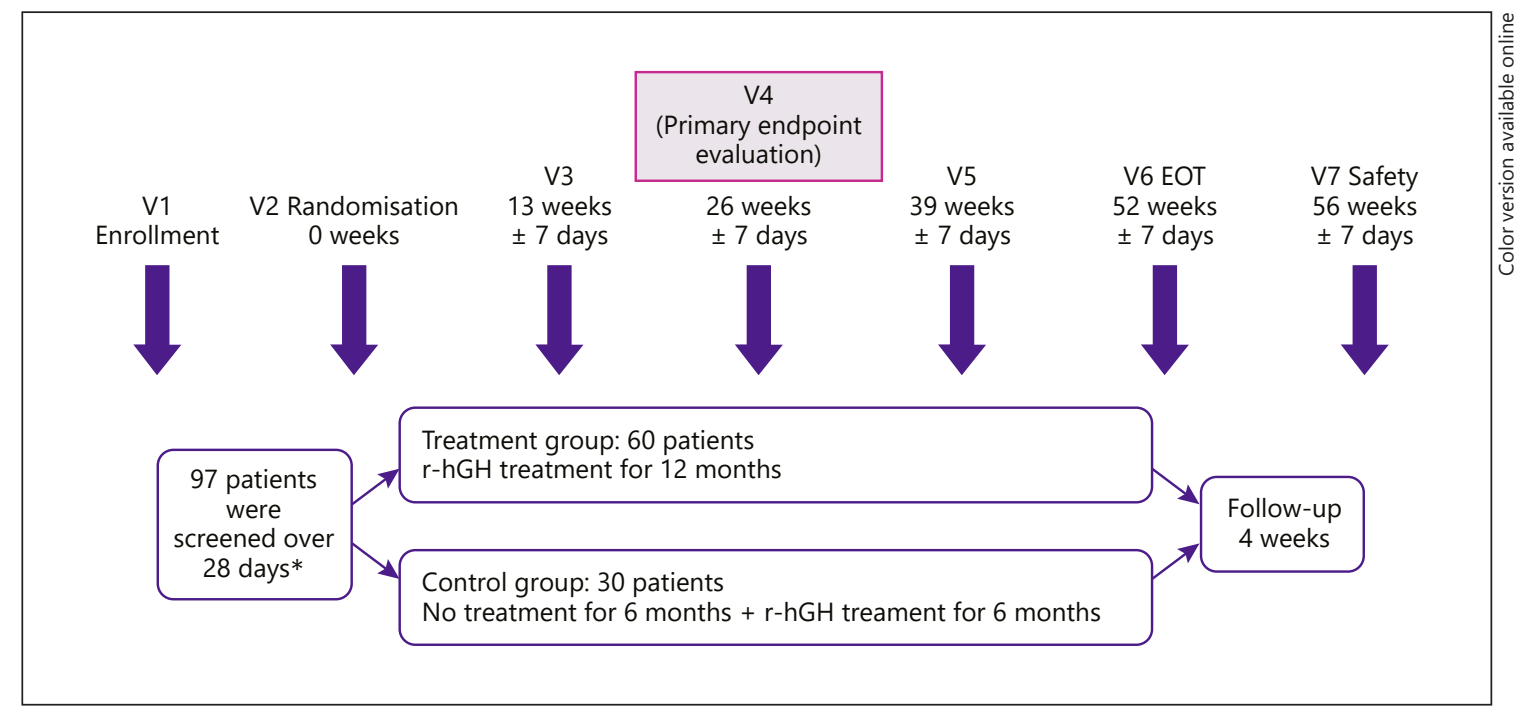

Fig. 1. SYNERGY study design. * Seven children did not meet the screening criteria; therefore, 90 patients were enrolled and randomised in the intention-to-treat population. EOT, end of treatment; $\mathrm{r}-\mathrm{hGH}$, recombinant human growth hormone.

Table 1. Demographics at baseline

\begin{tabular}{|c|c|c|}
\hline & $\begin{array}{l}\text { Treatment (r-hGH) group } \\
(n=59)\end{array}$ & $\begin{array}{l}\text { Control group } \\
(n=30)\end{array}$ \\
\hline Male/female & $30(51 \%) / 29(49 \%)$ & $17(57 \%) / 13(43 \%)$ \\
\hline Chronological age, years & $6.79(1.54)$ & $6.83(1.61)$ \\
\hline Height $^{1}, \mathrm{~cm}$ & $108.21(7.72)$ & $108.9(8.34)$ \\
\hline Height SDS & $-2.26(0.37)$ & $-2.33(0.34)$ \\
\hline Weight, kg & $18.09(3.43)$ & $18.24(3.11)$ \\
\hline Weight SDS & $-1.95(0.96)$ & $-1.85(1.05)$ \\
\hline BMI SDS & $-0.55(0.97)$ & $-0.37(1.12)$ \\
\hline Birth weight, kg & $2.95(0.38)$ & $3.04(0.30)$ \\
\hline Height velocity, $\mathrm{cm} /$ year & $5.65(1.65)$ & $4.98(1.95)$ \\
\hline Bone age $^{2}$, years & $5.21(1.56)$ & $5.04(1.56)$ \\
\hline Ratio of bone age to chronological age & $0.76(0.13)$ & $0.73(0.11)$ \\
\hline Target height ${ }^{3}, \mathrm{~cm}$ & $162.93(7.20)$ & $162.80(6.66)$ \\
\hline Target height ${ }^{4}$ SDS & $-0.59(0.48)$ & $-0.70(0.45)$ \\
\hline Peak GH, $\mu \mathrm{g} / \mathrm{L}$ & $20.01(14.24)$ & $22.25(12.51)$ \\
\hline Serum IGF-1, $\mu \mathrm{g} / \mathrm{L}$ & $113.56(42.81)$ & $125.01(65.68)$ \\
\hline Serum IGF-1 SDS & $-0.60(0.99)$ & $-0.52(1.33)$ \\
\hline Serum IGFBP-3, $\mu \mathrm{g} / \mathrm{L}$ & $3,838.98(814.25)$ & $3,935.67(866.42)$ \\
\hline Serum IGFBP-3 SDS & $0.43(0.57)$ & $0.52(0.57)$ \\
\hline
\end{tabular}

Data are $n(\%)$ or mean (SD). Data are reported in the safety population (treatment $n=59$ [ 1 child did not receive the study drug and was excluded]). BMI, body mass index; GH, growth hormone; IGF, insulin-like growth factor; IGFBP-3, insulin-like growth factor binding protein 3; r-hGH: recombinant human growth hormone; SD, standard deviation; SDS, standard deviation score.

${ }^{1}$ Mean of 2-3 measurements per child. ${ }^{2}$ Bone age calculated from radiographs of the wrist assessed by 2 investigators according to the standards of Greulich and Pyle [14]. ${ }^{3}$ Target height was estimated from sex-adjusted midparental height (father's height - mother's height) $/ 2$; gender adjustment was achieved by adding $6.5 \mathrm{~cm}$ for boys and subtracting $6.5 \mathrm{~cm}$ for girls [30]. ${ }^{4}$ Target height SDS was calculated as 0.72 multiplied by (father's height SDS - mother's height SDS)/2 [31]. 


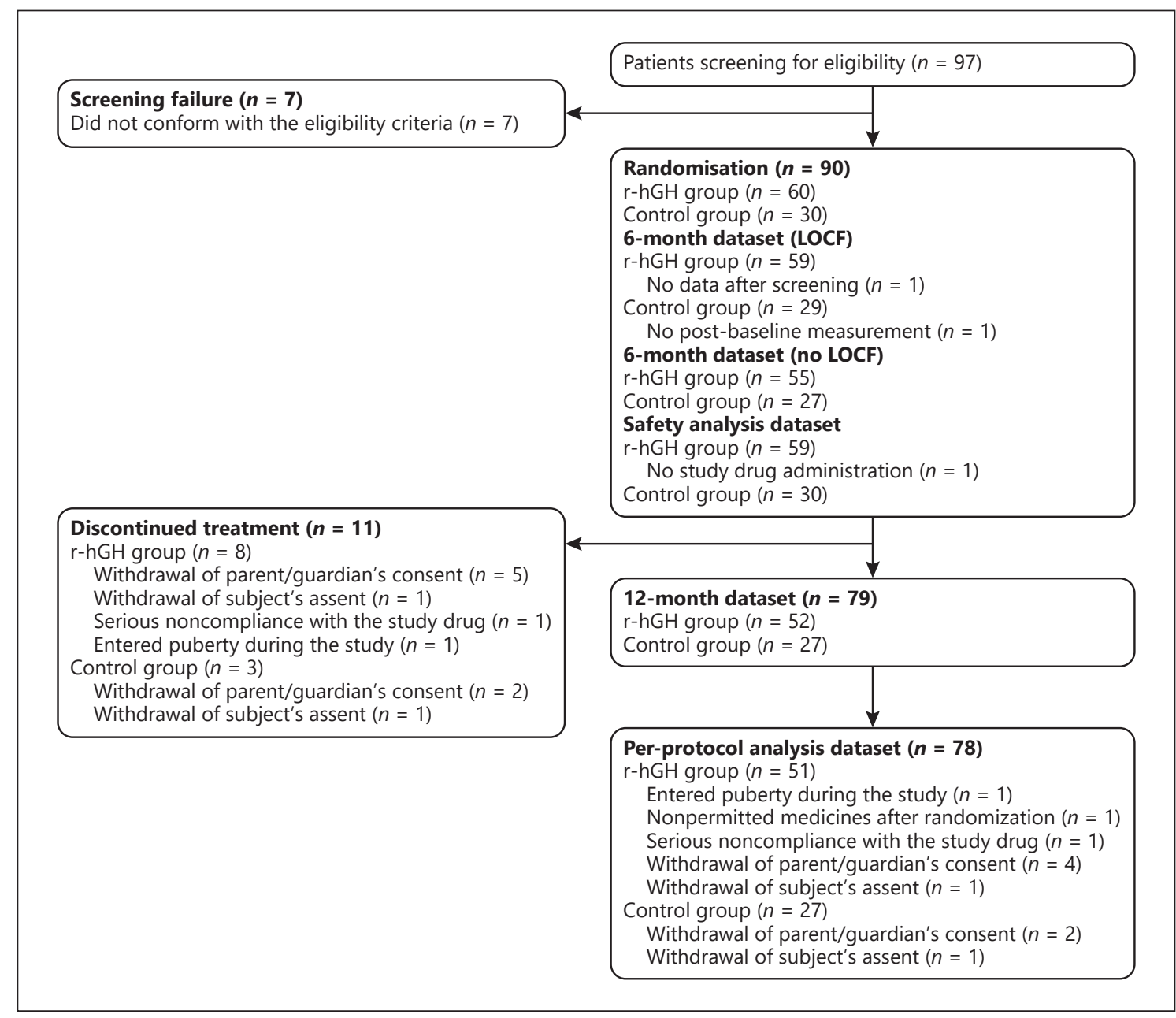

Fig. 2. Flow of children through the study. LOCF, last observation carried forward; $\mathrm{r}-\mathrm{hGH}$, recombinant human growth hormone.

verified by a second investigator at a different centre. Routine serological and haematological tests were done at screening and at week 26 and week 52. IGF-1, IGFBP-3, HbA1c, and thyroid function tests were done at the screening visit and at weeks 13 (treatment group only), 26, 39, and 52. Serum IGF-1 and IGFBP-3 concentrations were assessed at an independent central laboratory (Seoul Medical Science Institute, Seoul, South Korea) using commercial assays (Immulite, Siemens Healthcare Korea, Seoul, South Korea). Sexadjusted and age-adjusted IGF-1 SDS and IGFBP-3 SDS were calculated using commercial software (SDSeasy, Medidiagnost, Reutlingen, Germany). Cross-calibration of the assays showed that data from the Immulite IGF-1 assay could be used directly to calculate the IGF-1 SDS, whereas data from the Immulite IGFBP-3 assay required conversion using the following formula: IGFBP-3 $($ Mediagnost $)=$ IGFBP-3 $($ Immulite $) \times 0.3644+1.392$.

\section{Statistical Analyses}

72 children with ISS were estimated to provide $>90 \%$ power to detect a statistically significant difference in height velocity, ex- pecting a difference of $2 \mathrm{~cm} /$ year between groups after 6 months. To achieve this number, an estimated 87 children with ISS were required for randomisation. Only the primary endpoint was used to determine statistical significance of the treatment effect. All other $p$ values are presented for information only.

The primary endpoint was analysed in the intention-to-treat (ITT) population (all children who were randomly assigned to a treatment group and had at least 1 post-baseline height measurement) using two-sided analysis of covariance (ANCOVA), adjusted for baseline age and height as covariates and with last observation carried forward (LOCF). Between-group differences are leastsquares mean differences. Sensitivity analyses of the primary endpoint were done in the ITT population without LOCF and in the children who completed the study (per-protocol population).

Analyses of the secondary endpoints were exploratory and reported in children in the ITT population who completed the study. Only available data were used in the analyses of the secondary endpoints. Safety outcomes were reported in children who received at least 1 dose of $\mathrm{r}-\mathrm{hGH}$ and had post-baseline safety data. The oc- 
Fig. 3. Change in height velocity at 6 months. Data are mean (SD); only positive $\mathrm{SD}$ are shown. $p$ values are for withingroup comparisons. Intention-to-treat with LOCF. Treatment group: $n=59$ (1 patient was excluded from the baseline and change from baseline analyses owing to no height value recorded for 6 months prior to screening; this patient withdrew and there were no data after visit 1$)$. Control group: $n=29$ (6-month height measurements were missing for 3 children; these were replaced by measurements at withdrawal for 2 children; 1 child had no post-baseline measurement and, therefore, was not included in the analysis). LOCF, last observation carried forward; $\mathrm{r}-\mathrm{hGH}$, recombinant human growth hormone; SD, standard deviation.

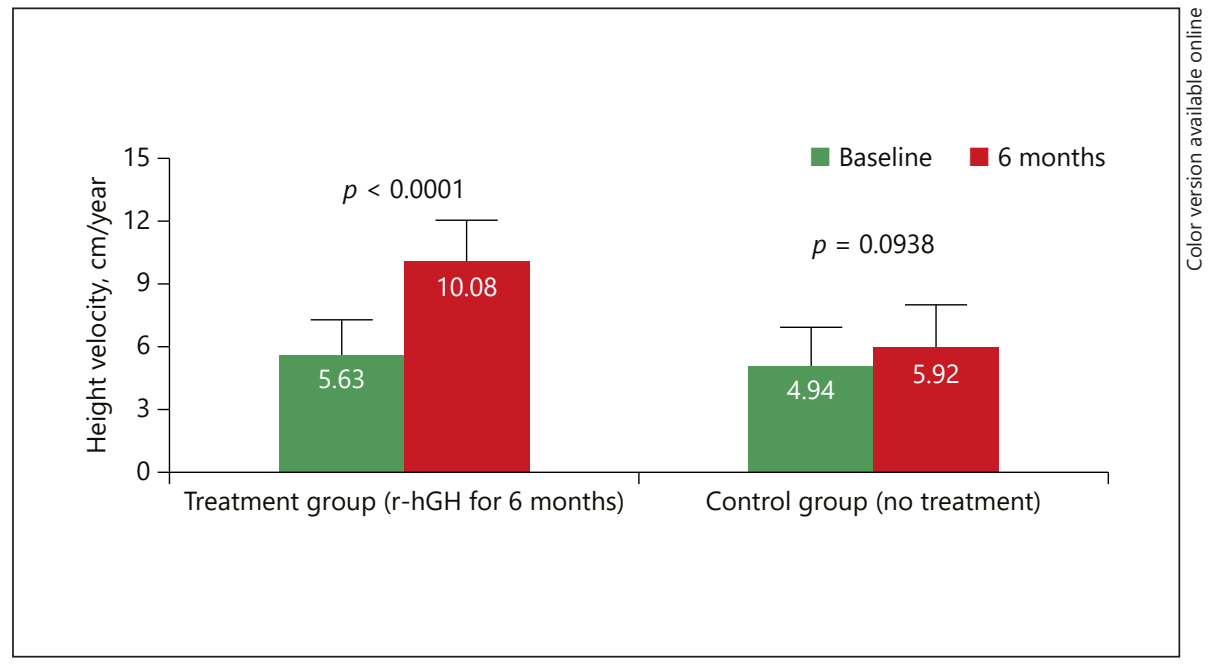

currence of AEs and serious AEs, according to entries in the treatment diaries, was compared using Fisher's test. Adherence was calculated from the proportion of prescribed doses administered according to drug diaries. Nonadherence was defined as receipt of $<75 \%$ of expected injections.

\section{Results}

\section{Children}

97 children with ISS were enrolled and screened across the 9 study sites in South Korea. Of these, 90 children met the eligibility criteria and were randomised (60 to the treatment group and 30 to the control group; ITT population [Fig. 1]).

Demographics were similar between the two groups (Table 1). The flow of children through the study is shown in Figure 2. 89 children in the ITT population received $\geq 1$ dose of $\mathrm{r}-\mathrm{hGH}$ (safety population). Analysis of the primary endpoint was done in the ITT LOCF population (59 children in the treatment group [ 1 child had no data after screening] and 29 children in the control group [1 child had no post-baseline measurements]; Fig. 2). Six of these children ( 4 in the treatment group and 2 in the control group) were missing measurements at 6 months; the 6-month values for these children were reported as LOCF. One subject who was born at a gestational age of 30 weeks was enrolled in the study before the protocol was amended to only include subjects $>34$ weeks of gestation; however, this subject was judged to have a normal weight $(1.5 \mathrm{~kg})$ based on gestational age and was included in the analyses. 79 children completed the study to 12 months, 78 without any protocol deviations (per-protocol population).

\section{Primary Endpoint}

The mean increase $( \pm S D)$ in height velocity from baseline to 6 months was significant in the treatment group (from $5.63 \mathrm{~cm} /$ year [1.62] to $10.08 \mathrm{~cm} /$ year [1.92]; $p<$ 0.0001 ) but not in the control group (from $4.94 \mathrm{~cm} /$ year [1.91] to $5.92 \mathrm{~cm} /$ year [2.01]; $p=0.0938)$. The betweengroup difference was $3.47 \mathrm{~cm} /$ year $(95 \%$ confidence interval [CI] 2.17-4.78; $p<0.0001$ by ANCOVA); this between-group increase was clinically relevant (Fig. 3). Sensitivity analyses gave similar between-group differences to the LOCF analysis in both the ITT population without LOCF (3.75 cm/year, 95\% CI 2.51-5.00; $p<0.0001$ by ANCOVA) and in the per-protocol population $(3.85 \mathrm{~cm} /$ year, 95\% CI 2.58-5.12; $p<0.0001$ by ANCOVA).

\section{Secondary Endpoints}

Adherence was $>90 \%$ throughout the study (treatment group: $94.38 \%$ at 6 months and $93.27 \%$ at 12 months; control group: $95.69 \%$ at 12 months).

The mean increase $( \pm S D)$ in height velocity from baseline at 12 months was $3.77 \mathrm{~cm} /$ year (2.21) in the children who had received $\mathrm{r}-\mathrm{hGH}$ for 12 months (treatment group) and $2.86 \mathrm{~cm} /$ year $(1.95)$ in the children who had received r-hGH for only the last 6 months of the study (control group). The between-group difference at 12 months was $0.96 \mathrm{~cm} /$ year $(95 \% \mathrm{CI}-0.04$ to $1.97, p=0.0598$ by ANCOVA).

Children in the treatment group had a statistically and clinically greater increase in mean height compared with those in the control group at 6 months (difference 2.27 $\mathrm{cm}, 95 \%$ CI $1.83-2.71 ; p<0.0001$ by ANCOVA) and 12 months (difference $1.50 \mathrm{~cm}, 95 \%$ CI $0.87-2.13 ; p<0.0001$
48

Horm Res Paediatr 2018;90:44-53 DOI: $10.1159 / 000491016$
Chung/Yoo/Hwang/Ko/Kim/Jin/Lee/ Han/Paranchothy/Suh 
Fig. 4. Change in height (a) and height SDS (b) at 6 and 12 months. Data are mean (SD); only positive SD are shown. Children completing the study at 12 months: treatment, $n=52$; control, $n=27$. Reasons for discontinuation in the treatment group: 5 parents/guardians withdrew consent; 1 child withdrew consent; 1 child had serious noncompliance with the study drug; 1 girl entered puberty (breast Tanner stage $\geq 2$ ). Reasons for discontinuation in the control group: 2 parents/guardians withdrew consent; 1 child withdrew consent. SD, standard deviation; SDS, standard deviation score.

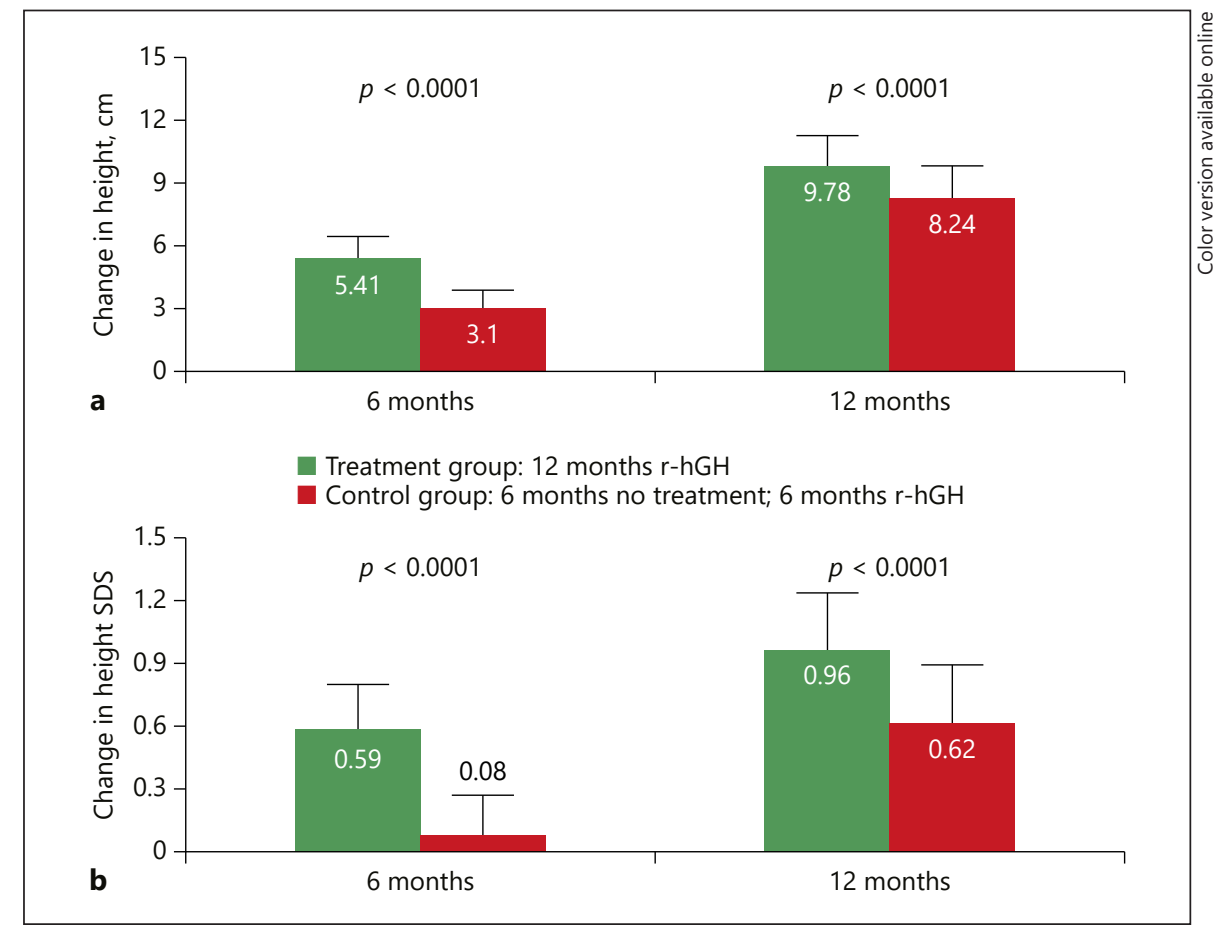

Table 2. Safety outcomes: treatment-emergent adverse events

\begin{tabular}{lllll}
\multicolumn{2}{r}{ r-hGH 12 months $(n=59)$} & & Control $(n=30)$ & \\
\cline { 1 - 1 } with event, & events, & & with event, & events, \\
$n(\%)$ & $n$ & & $n(\%)$ & $n$
\end{tabular}

12 months of treatment (baseline to last visit)

Any TEAE

Any serious TEAE

Any treatment-related TEAE

Any treatment-related serious TEAE

Any TEAE leading to study treatment discontinuation

Any TEAE leading to death

$42(71.2)$
$3(5.1)$
$0(0)$
$0(0)$
$0(0)$
$0(0)$

\begin{tabular}{rcr}
179 & $18(60.0)$ & 83 \\
5 & $1(3.3)$ & 3 \\
0 & $1(3.3)$ & 2 \\
0 & $0(0)$ & 0 \\
0 & $0(0)$ & 0 \\
0 & $0(0)$ & 0 \\
\hline
\end{tabular}

6 months of treatment (baseline to 6-month visit)
Any TEAE
Any serious TEAE
Any treatment-related TEAE
Any treatment-related serious TEAE
Any TEAE leading to study treatment discontinuation
Any TEAE leading to death

$38(64.4)$
$3(5.1)$
$0(0)$
$0(0)$
$\mathrm{n} / \mathrm{a}$
$0(0)$

93
5
0
0
$\mathrm{n} / \mathrm{a}$
0

$15(50.0)$
$1(3.3)$
$0(0)$
$0(0)$
$\mathrm{n} / \mathrm{a}$
$0(0)$

r-hGH, recombinant human growth hormone; TEAE, treatment-emergent adverse event; n/a, not applicable.

by ANCOVA) (Fig. 4). The mean change in height SDS was also statistically and clinically greater in the treatment group than in the control group at 6 months (difference $0.51,95 \%$ CI $0.42-0.60 ; p<0.0001$ by ANCOVA) and at 12 months (difference $0.33,95 \%$ CI $0.21-0.45 ; p<$ 0.0001 by ANCOVA) (Fig. 4).

At baseline, there were no differences between groups in age-adjusted and sex-adjusted serum IGF-1 SDS 
$(-0.6 \pm 1.0$ vs. $-0.5 \pm 1.3)$ and IGFBP-3 SDS $(0.4 \pm 0.6$ vs. $0.5 \pm 0.6$ ) (Table 1). In the control group, mean IGF-1 SDS and mean IGFBP-3 SDS did not change during the first 6 months but increased after treatment with r-hGH started (IGF-1 SDS: from $-0.5 \pm 1.2$ to $1.1 \pm 1.3$; IGFBP-3 SDS: from $0.4 \pm 0.5$ to $0.6 \pm 0.5$ ). In the treatment group, IGF-1 SDS increased to $1.0 \pm 1.2$ after 6 months and $1.3 \pm 1.1$ after 12 months, and IGFBP-3 SDS increased to $0.8 \pm 0.5$ after 6 months and $0.8 \pm 0.5$ after 12 months (Fig. 5). The mean between-group increase at 6 months, with baseline SDS (IGF-1 or IGFBP-3, as appropriate) and age as covariates, was significant for both biomarkers $(p<0.0001)$. Throughout $\mathrm{r}-\mathrm{hGH}$ treatment, the mean IGF-1 SDS and IGFBP-3 SDS values were within the upper normal range. At 12 months, IGF-1 SDS was $>2$ in 19 of $79(24 \%)$ patients. At 12 months, IGF-1 SDS was $>3$ in 4 of 79 (5\%) patients, all of whom had IGFBP-3 SDS $>1.2$.

In a post hoc analysis, no differences in BMI SDS were seen between the treatment and control groups at 6 months (least-squares mean difference between groups $-0.15,95 \%$ CI -0.42 to 0.12 ; $p=0.8513$ by ANCOVA) or at 12 months $(-0.15,95 \% \mathrm{CI}-0.39$ to $0.09 ; p=0.4854$ by ANCOVA).

AEs were reported in $42(71 \%)$ children (179 events) in the treatment group and 18 (60\%) children (83 events) in the control group throughout the full 12-month period (Table 2). The most common AEs reported in both groups were nasopharyngitis (24 children [41\%] in the treatment group and 9 children [30\%] in the control group), upper respiratory tract infection (14 children [24\%] in the treatment group and 9 children [30\%] in the control group) and pyrexia (4 children [7\%] in the treatment group and 3 children [10\%] in the control group). Five serious AEs were reported for 3 children in the treatment group ( 1 chronic tonsillitis, 2 adenoidal hypertrophy, 1 tonsillar hypertrophy, and 1 croup infectious) and 3 serious AEs ( 1 chronic sinusitis, 1 pyrexia, and $1 \mathrm{~T}$-cell lymphoma) were reported for 1 child in the control group. This child experienced $\mathrm{T}$-cell lymphoma during the nontreatment period and had not been exposed to $r-$ hGH during the whole study period. None of the serious AEs were considered by the study investigators to be treatment related.

Two AEs (hypothyroidism and headache) in 1 child in the control group during the treatment period in the second 6 months of the study were possibly related to the study treatment. No clinically significant changes in laboratory values, physical examinations, or vital signs were observed and no deaths or AEs leading to treatment withdrawal were reported.

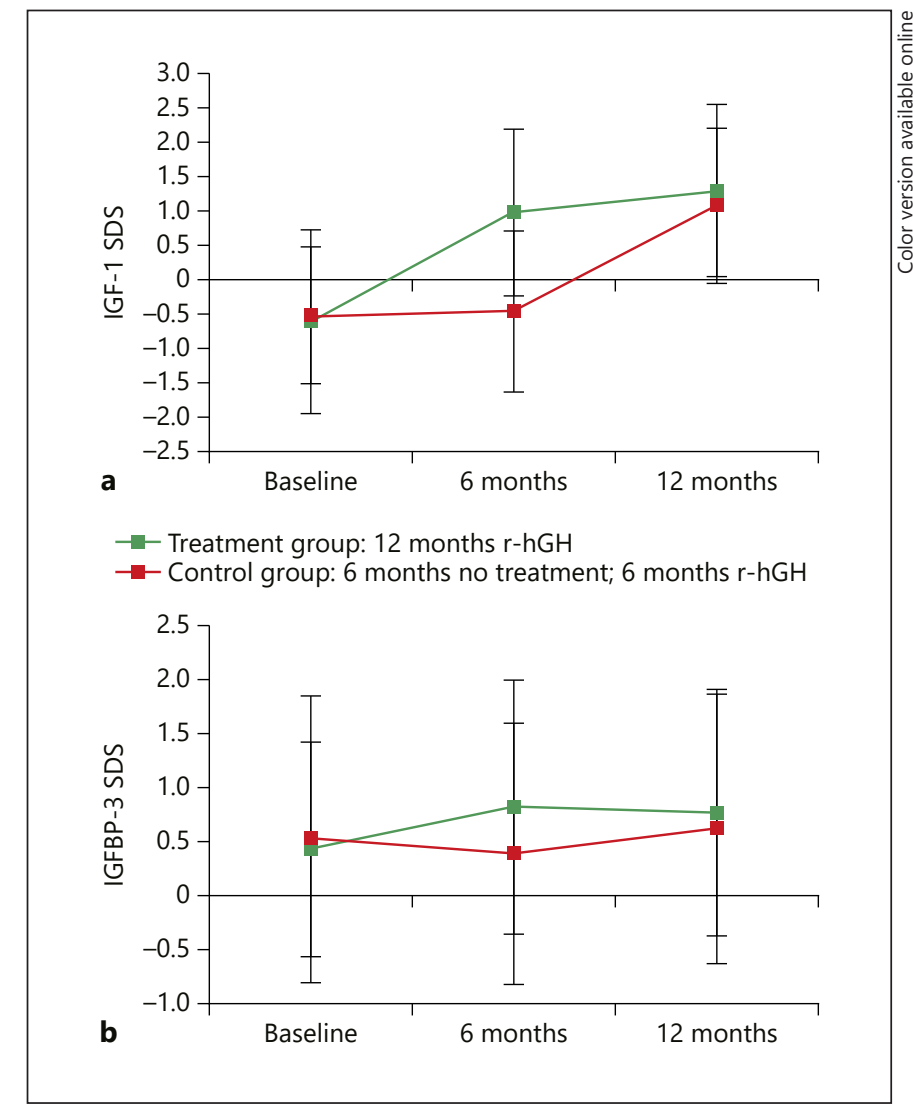

Fig. 5. Change in IGF-1 SDS (a) and IGFBP-3 SDS (b) from baseline at 6 months and 12 months. Data are mean (SD). Children completing the study at 12 months: treatment, $n=52$; control, $n=$ 27. Reasons for discontinuation in the treatment group: 5 parents/ guardians withdrew consent, 1 child withdrew consent, 1 child had serious noncompliance with the study drug, and 1 girl entered puberty (breast Tanner stage $\geq 2$ ). Reasons for discontinuation in the control group: 2 parents/guardians withdrew consent and 1 child withdrew consent. SD, standard deviation; SDS, standard deviation score.

\section{Discussion}

The results reported in this publication are from the first randomised clinical study (SYNERGY) on the shortterm height outcomes of $\mathrm{r}$-hGH in prepubertal Korean children with ISS. Several studies have previously been conducted on $\mathrm{r}-\mathrm{hGH}$ treatment in mixed populations of children with ISS across other regions [12]. However, only a few of these were randomised nontreatment controlled trials in prepubertal children that reported on short-term (up to 12 months) improvements in height outcomes, as are reported in this paper [15-18]. Furthermore, the parallel group design and the large number of 
well-defined children reported here make this study one of only a few that provide an objective assessment of $r$ $\mathrm{hGH}$ treatment in a population that is representative of children with ISS. The population reported here will most probably comprise the target population for this indication.

The results of the SYNERGY study show that Korean children treated with $\mathrm{r}-\mathrm{hGH}$ had clinically relevant and statistically significant increases in height velocity over 6 months, and continuous treatment led to clinically relevant growth enhancement for up to 12 months. The mean change in height SDS was statistically significant and clinically relevant at 6 months but not at 12 months, which is consistent with other clinical studies of $\mathrm{r}-\mathrm{hGH}$ in children with ISS. The safety profile of $\mathrm{r}-\mathrm{hGH}$ treatment in this new indication was comparable to the already approved indications. Although our data cannot be extrapolated to long-term height gains, the short-term responses to $\mathrm{r}$ hGH in the first 6-12 months of treatment have correlated with long-term efficacy. This finding has been reported in prediction models based on large cohorts treated in standard clinical practice whose progress is followed in observational databases $[13,19,20]$. Furthermore, in one published randomised controlled trial of somatropin treatment versus nontreatment in a small cohort of short girls who were prepubertal at the start of treatment, favourable gains in near-final height were achieved over a mean period of 6.2 years of treatment, with no untoward effect on pubertal progression [21]. In accordance with these data, there is good reason to expect that the children treated with $\mathrm{r}-\mathrm{hGH}$ in the SYNERGY study are likely to have a good chance of reaching an improved near-final height.

Despite the paucity of available diagnostic tests for ISS, height deficits will already be evident in children by the time they start school. This means that investigations and the commencement of treatment can usually be achieved at an early age, giving a child the best chance of achieving the most favourable height outcomes as an adult $[4,12,22-24]$. Older children, including those who have started pubertal development, may still also benefit from treatment with $\mathrm{r}-\mathrm{hGH}$. To remove the confounding effect of the pubertal growth spurt on the short-term growth outcomes, we included only younger children who had not entered puberty in the SYNERGY study. There are several published trials that report favourable final-height outcomes following treatment in pubertal or peripubertal children [21, 23, 25, 26]. Furthermore, although the children in the control group were still prepubescent during the second phase of the study, these

Growth Hormone in Korean Children with ISS children achieved a height velocity that was similar to that achieved in the treatment group after 6 months of treatment with $\mathrm{r}-\mathrm{hGH}$. This finding further supports the clinical relevance of the treatment effect and possibly provides evidence of catch-up growth response to r-hGH in older children.

Based on previously published data reporting on dosedependent effects on first-year height velocity and subsequent gain in adult height $[23,24]$, a dose of $0.067 \mathrm{mg} / \mathrm{kg}$ for 6 days per week $(0.4 \mathrm{mg} / \mathrm{kg} /$ week $)$ was chosen as the dose most likely to give an optimum response over the initial 6 months. This dose is within the generally accepted range of doses used for the treatment of children who do not have GH deficiency [6].

After the start of high-dose r-hGH therapy, supra-normal IGF- 1 concentrations ( $>2$ SDS) were observed in $24 \%$ of patients; this is comparable to the proportion of patients with supra-normal IGF-1 concentrations in other studies of high-dose r-hGH treatment [27]. Increased IGF-1 concentrations may be cause for concern, on the basis of epidemiological studies in healthy adults that showed a slight association between high IGF-1 and the development of some forms of cancer [28]. However, the relevance of such studies for paediatric patients treated with $\mathrm{r}-\mathrm{hGH}$ has not been established, according to the consensus statement of paediatric societies [29].

There are some limitations that must be taken into account when interpreting the results of this study. In studies of children with ISS, the cohorts are commonly heterogeneous regarding the causes of short stature. Furthermore, treatment responses with $\mathrm{r}-\mathrm{hGH}$ may fluctuate in clinical practice, even though the population reported here was considered to be representative of children with ISS who would receive treatment with $\mathrm{r}-\mathrm{hGH}$. Although this study was adequately powered, the population was still small. To present the full picture of the effect of treatment length and timing on the final height that is achieved in this population, a greater number of children would need to be treated with $\mathrm{r}-\mathrm{hGH}$ and followed up for longer. Finally, although the trends reported here are in line with those reported for final height, the effect on final height after a longer duration of treatment has been reported for several studies $[13,21,23,25]$.

In conclusion, continuous treatment with $\mathrm{r}-\mathrm{hGH}$ in the SYNERGY study demonstrated clinically relevant and statistically significant growth enhancement after 6 months, which was maintained over 12 months of continuous treatment. Despite a delayed start to treatment, children in the control group showed catch-up growth in response to 6 months of treatment that was comparable 
to that seen in the treatment group after the first 6 months of treatment. The safety profile for the SYNERGY study was consistent with the known profile of $\mathrm{r}-\mathrm{hGH}$ and no new safety concerns were reported for the children with ISS treated with $\mathrm{r}-\mathrm{hGH}$.

\section{Acknowledgements}

The authors would like to thank all the children and their parents and guardians who took part in the study. They would also like to thank all the investigators who contributed to the SYNERGY study. The authors would particularly like to thank Charmain Quigley (Sydney Children's Hospital, Sydney, Australia), Ekaterina Koledova (Merck KGaA, Darmstadt, Germany), and Werner Blum (Children's Hospital, University of Giessen, Giessen, Germany) for their contributions to the SYNERGY study.

The study was sponsored by Merck Ltd, Korea, an affiliate of Merck KGaA, Darmstadt, Germany. Medical writing support was provided by Steven Goodrick, inScience Communications, UK, funded by Merck KGaA, Darmstadt, Germany.

\section{Disclosure Statement}

W.Y.C. has served as a consultant to LG. H.-W.Y. has served on advisory boards for Merck KGaA and Sanofi-Genzyme and has received speaker honoraria from Sanofi-Genzyme. J.S.H. has no disclosures to declare. C.W.K. has served as a consultant to LG, Dong- $\mathrm{A}$, and Takeda and has received speaker honoraria from Novo Nordisk. H.-S.K. has served as a consultant to LG. D.-K.J. has no disclosures to declare. K.-H.L. has served as a consultant to LG and Novo Nordisk. H.-S.H. has served as a consultant to LG and Dong-A. B.-K.S. has served as a consultant to LG. At the time of the study, P.P. was an employee of Merck Ltd, Korea, an affiliate of Merck KGaA, Darmstadt, Germany.

\section{Author Contributions}

J.S.H. and B.-K.S. contributed to the development of the study concept, the design of the study, the interpretation of the data, and revision of the manuscript. W.Y.C., H.-W.Y., C.W.K., H.-S.K., D.-K.J., K.-H.L., H.-S.H., and P.P. contributed to the writing and review of the manuscript. All authors have read and approved the final version of the manuscript.

\section{References}

1 American Academy of Pediatrics Committee on Drugs and Committee on Bioethics. Considerations related to the use of recombinant human growth hormone in children. Pediatrics. 1997 Jan;99(1):122-9.

2 Gharib H, Cook DM, Saenger PH, Bengtsson BA, Feld S, Nippoldt TB, et al.; American Association of Clinical Endocrinologists Growth Hormone Task Force. American Association of Clinical Endocrinologists medical guidelines for clinical practice for growth hormone use in adults and children-2003 update. Endocr Pract. 2003 Jan-Feb;9(1):64-76.

3 Growth Hormone Research Society; GH Research Society. Consensus guidelines for the diagnosis and treatment of growth hormone $(\mathrm{GH})$ deficiency in childhood and adolescence: summary statement of the GH Research Society. J Clin Endocrinol Metab. 2000 Nov;85(11):3990-3.

4 Wit JM, Clayton PE, Rogol AD, Savage MO, Saenger PH, Cohen P. Idiopathic short stature: definition, epidemiology, and diagnostic evaluation. Growth Horm IGF Res. 2008 Apr; 18(2):89-110.

5 Savage MO, Burren CP, Rosenfeld RG. The continuum of growth hormone-IGF-I axis defects causing short stature: diagnostic and therapeutic challenges. Clin Endocrinol (Oxf). 2010 Jun;72(6):721-8.

6 Cohen P, Rogol AD, Deal CL, Saenger P, Reiter EO, Ross JL, et al.; 2007 ISS Consensus Workshop participants. Consensus statement on the diagnosis and treatment of children with idiopathic short stature: a summary of the Growth Hormone Research Society, the Lawson Wilkins Pediatric Endocrine Society, and the European Society for Paediatric Endocrinology Workshop. J Clin Endocrinol Metab. 2008 Nov;93(11):4210-7.

7 Wit JM, Kamp GA, Rikken B. Spontaneous growth and response to growth hormone treatment in children with growth hormone deficiency and idiopathic short stature. Pediatr Res. 1996 Feb;39(2):295-302.

8 Buchlis JG, Irizarry L, Crotzer BC, Shine BJ, Allen L, MacGillivray MH. Comparison of final heights of growth hormone-treated vs. untreated children with idiopathic growth failure. J Clin Endocrinol Metab. 1998 Apr; 83(4):1075-9.

9 Sheiner E, Levy A, Katz M, Mazor M. Short stature-an independent risk factor for Cesarean delivery. Eur J Obstet Gynecol Reprod Biol. 2005 Jun;120(2):175-8.

10 Price DA. Spontaneous adult height in patients with idiopathic short stature. Horm Res. 1996;45 Suppl 2:59-63.

11 Magnusson PK, Gunnell D, Tynelius P, Davey Smith G, Rasmussen F. Strong inverse association between height and suicide in a large cohort of Swedish men: evidence of early life origins of suicidal behavior? Am J Psychiatry. 2005 Jul;162(7):1373-5.

12 Quigley CA. Growth hormone treatment of non-growth hormone-deficient growth disorders. Endocrinol Metab Clin North Am. 2007 Mar;36(1):131-86.
13 Ranke MB, Lindberg A, Price DA, Darendeliler F, Albertsson-Wikland K, Wilton P, et al.; KIGS International Board. Age at growth hormone therapy start and first-year responsiveness to growth hormone are major determinants of height outcome in idiopathic short stature. Horm Res. 2007;68(2):53-62.

14 Greulich WW, Pyle SI. Radiographic Atlas of Skeletal Development of the Hand and Wrist, 2nd ed. Stanford: Stanford University Press; 1959.

15 Genentech Collaborative Study Group. Idiopathic short stature: results of a one-year controlled study of human growth hormone treatment. J Pediatr. 1989 Nov;115(5 Pt 1):713-9.

16 Cowell CT; Australasian Paediatric Endocrine Group. Effects of growth hormone in short, slowly growing children without growth hormone deficiency. Acta Paediatr Scand Suppl. 1990;366 s366:29-30.

17 McCaughey ES, Mulligan J, Voss LD, Betts PR. Growth and metabolic consequences of growth hormone treatment in prepubertal short normal children. Arch Dis Child. 1994 Sep;71(3):201-6.

18 Soliman AT, abdul Khadir MM. Growth parameters and predictors of growth in short children with and without growth hormone (GH) deficiency treated with human GH: a randomized controlled study. J Trop Pediatr. 1996 Oct;42(5):281-6.

19 Zucchini S. Growth hormone use in the treatment of idiopathic short stature. Curr Opin Investig Drugs. 2008 Apr;9(4):396-401. 
20 Bakker B, Frane J, Anhalt H, Lippe B, Rosenfeld RG. Height velocity targets from the national cooperative growth study for first-year growth hormone responses in short children. J Clin Endocrinol Metab. 2008 Feb;93(2): 352-7.

21 McCaughey ES, Mulligan J, Voss LD, Betts PR. Randomised trial of growth hormone in short normal girls. Lancet. 1998 Mar; 351(9107):940-4.

22 Bryant J, Baxter L, Cave CB, Milne R. Recombinant growth hormone for idiopathic short stature in children and adolescents. Cochrane Database Syst Rev. 2007 Jul;(3):CD004440.

23 Albertsson-Wikland K, Aronson AS, Gustafsson J, Hagenäs L, Ivarsson SA, Jonsson B, et al. Dose-dependent effect of growth hormone on final height in children with short stature without growth hormone deficiency. J Clin Endocrinol Metab. 2008 Nov;93(11):434250.

24 Wit JM, Rekers-Mombarg LT, Cutler GB Jr, Crowe B, Beck TJ, Roberts K, et al. Growth hormone $(\mathrm{GH})$ treatment to final height in children with idiopathic short stature: evidence for a dose effect. J Pediatr. 2005 Jan; 146(1):45-53.

25 Leschek EW, Rose SR, Yanovski JA, Troendle JF, Quigley CA, Chipman JJ, et al.; National Institute of Child Health and Human Development-Eli Lilly \& Co. Growth Hormone Collaborative Group. Effect of growth hormone treatment on adult height in peripubertal children with idiopathic short stature: a randomized, double-blind, placebo-controlled trial. J Clin Endocrinol Metab. 2004 Jul;89(7):3140-8.

26 Volta C, Bernasconi S, Tondi P, Salvioli V, Ghizzoni L, Baldini A, et al. Combined treatment with growth hormone and luteinizing hormone releasing hormone-analogue (LHRHa) of pubertal children with familial short stature. J Endocrinol Invest. 1993 Nov; 16(10):763-7.

27 Juul A. The role of insulin-like growth factors in growth hormone deficiency. In: Ranke MB,
Price DA, Reiter EO, editors. Growth Hormone Therapy in Pediatrics: 20 Years of KIGS. Basel: Karger; 2007. pp. 70-82.

28 Renehan AG, Zwahlen M, Minder C, O'Dwyer ST, Shalet SM, Egger M. Insulin-like growth factor (IGF)-I, IGF binding protein-3, and cancer risk: systematic review and meta-regression analysis. Lancet. 2004 Apr;363(9418): 1346-53.

29 Allen DB, Backeljauw P, Bidlingmaier M, Biller BM, Boguszewski M, Burman P, et al. GH safety workshop position paper: a critical appraisal of recombinant human GH therapy in children and adults. Eur J Endocrinol. 2016 Feb;174(2):1-9.

30 Chae HW, Suh I, Kwon AR, Kim YJ, Kim YH, Kang DR, et al. Longitudinal standards for height and height velocity in Korean children and adolescents: the Kangwha study [corrected]. J Korean Med Sci. 2013 Oct;28(10):1512-7.

31 Hermanussen $M$, Cole J. The calculation of target height reconsidered. Horm Res. 2003; 59(4):180-3. 